\title{
Correlation of Neuroimaging Findings with Clinical Presentation and Laboratory Data in Patients with COVID-19: A Single-Center Study
}

\author{
Seyhmus Kavak (D), ${ }^{1}$ Mehmet Serdar Yildirim, ${ }^{2}$ Rojhat Altındag, ${ }^{3}$ Yilmaz Mertsoy, ${ }^{4}$ \\ Mehmet Fuat Alakus, ${ }^{5}$ Mehmet Diyaddin Guleken, ${ }^{6}$ and Safak Kaya ${ }^{7}$ \\ ${ }^{1}$ Department of Radiology, Health Sciences University, Gazi Yasargil Research and Training Hospital, Diyarbakir, Turkey \\ ${ }^{2}$ Department of Internal Medicine, Health Sciences University, Gazi Yasargil Research and Training Hospital, Diyarbakir, Turkey \\ ${ }^{3}$ Department of Cardiology, Health Sciences University, Gazi Yasargil Research and Training Hospital, Diyarbakir, Turkey \\ ${ }^{4}$ Department of Orthopedics and Traumatology, Health Sciences University, Gazi Yasargil Research and Training Hospital, \\ Diyarbakir, Turkey \\ ${ }^{5}$ Department of Ophthalmology, Health Sciences University, Gazi Yasargil Research and Training Hospital, Diyarbakir, Turkey \\ ${ }^{6}$ Department of Psychiatry, Health Sciences University, Gazi Yasargil Research and Training Hospital, Diyarbakir, Turkey \\ ${ }^{7}$ Depatment of Infectious Diseases and Clinical Microbiology, Health Sciences University, Gazi Yasargil Research and \\ Training Hospital, Diyarbakir, Turkey
}

Correspondence should be addressed to Seyhmus Kavak; s.ozgurkavak@hotmail.com

Received 1 May 2021; Revised 10 July 2021; Accepted 27 July 2021; Published 15 August 2021

Academic Editor: Hsian Min Chen

Copyright @ 2021 Seyhmus Kavak et al. This is an open access article distributed under the Creative Commons Attribution License, which permits unrestricted use, distribution, and reproduction in any medium, provided the original work is properly cited.

\begin{abstract}
Background. This study was aimed at revealing neuroimaging findings in COVID-19 patients and at discussing their relationship with epidemiological data and some laboratory parameters. Materials and Method. This study included 436 cases of COVID-19 and 40 cases of non-COVID-19 acute/subacute thromboembolism who underwent at least one neuroimaging procedure due to neurological symptoms between April 2020 and December 2020. The group of COVID-19-positive acute/subacute thromboembolism cases was compared with both the group of normal brain imaging cases and the non-COVID-19 acute/subacute thromboembolism group in terms of demographic data and laboratory parameters. Results. When the acute/subacute thromboembolism group and neuroimaging findings were compared in terms of negative group, presence of comorbid disease, D-dimer level, and lymphocyte count in COVID-19 patients, a statistically significant difference was found ( $p=0.047,0.014$, and $<0.001$, respectively). COVID-19-positive and COVID-19-negative acute/subacute thromboembolism cases that were compared in terms of gender, neuroimaging reason, C-reactive protein, D-dimer level and lymphocyte count, a statistically significant difference was found $(p=0.003,<0.001,0.005,0.02$, and $<0.001$, respectively). Conclusion. Acute thromboembolic events are common in patients with COVID-19 due to a potentially increased procoagulant process. Neurological evaluation and, if necessary, detailed neuroimaging should be performed, especially in cases with high D-dimer levels.
\end{abstract}

\section{Introduction}

After the novel coronavirus (nCoV) first appeared in Wuhan, China, in December 2019, it spread rapidly, causing a pandemic shortly thereafter. This new virus was named severe acute respiratory syndrome coronavirus 2 (SARS-CoV-2), and the disease it caused was named "coronavirus disease 2019" (COVID-19) $[1,2]$. This virus exerts its effect by bind- ing with high affinity to angiotensin-converting enzyme 2 (ACE2) receptors in humans via virus spike proteins. These receptors are located in many organs of the body, such as the brain. In the brain, ACE2 receptors are widely expressed in glial cells and brainstem nuclei involved in the regulation of the cardiorespiratory system, the reticular activation system, and the motor cortex [3]. It is controversial whether high ACE2 receptor density, especially at the nucleus 
solitarius and nucleus ambiguus levels, contributes to severe respiratory dysfunction [4].

Two hypotheses have been proposed regarding how the virus accesses the central nervous system (CNS): the first is hematogenous spread of infected leukocytes through the compromised endothelial cells of the blood-brain barrier, similar to the spread of other viruses, and the second is retrograde spread along the axons of peripheral nerves, such as the olfactory nerve [5]. Once the virus reaches the CNS, it causes damage either by the cytokine storm that occurs or by direct action. In addition, it has been indicated that increased coagulation activity and a correlated increase in D-dimer level may cause secondary damage and increase the risk of thromboembolism [6-9].

COVID-19 can present with neurological symptoms such as anosmia, headache, impaired taste sensation, dizziness, syncope, and altered consciousness [10]. It has been reported in previous studies that thromboembolic ischemic and hemorrhagic strokes associated with COVID-19 have been observed in the early period. It has been suggested that conditions characterized by neurological sequelae, such as encephalitis and encephalopathy, ataxic seizures, GuillainBarré syndrome (GBS), demyelinating diseases, and neuromuscular disorders, may occur in the longer term [11-14].

The aim of this study is to discuss the relationship of brain imaging findings with laboratory and epidemiological data in patients with neurological symptoms followed up with the diagnosis of COVID-19. In addition, cases with acute/subacute thromboembolism without COVID-19 and cases with COVID-19-positive acute/subacute thromboembolism were compared.

\section{Materials and Method}

We retrospectively analyzed 5233 real-time reverse transcriptase-polymerase-chain reaction- (RT-PCR-) positive cases hospitalized in our hospital with a diagnosis of COVID-19 between April and December 2020 and included 436 cases who underwent at least one neuroimaging (Figure 1). Computed tomography (CT) and magnetic resonance $(\mathrm{MR})$ images of these patients were evaluated by 2 experienced radiologists (S.K. and R.D.). The opinion of a third radiologist (A.A.) was sought in cases of disagreement to achieve consensus. CT and MR images obtained during hospitalization or at the first admission to the hospital were examined for the presence of ischemia, infarction, bleeding, and encephalitis. The patient population was divided into 4 groups according to radiological data, and comparative statistical analyses of these groups were performed. The first group consisted of 46 patients with acute/subacute infarction with neuroimaging findings, and the second group consisted of the remaining 390 patients. Cases with nonspecific white matter changes on neuroimaging $(N=189)$ constituted the third group, and the remaining 247 cases constituted the fourth group. In addition, neuroimaging findings, clinical, epidemiological, and laboratory data of 40 patients diagnosed with non-COVID acute/subacute thromboembolism who were hospitalized in our hospital during the same period were analyzed and compared with group 1 . Laboratory tests

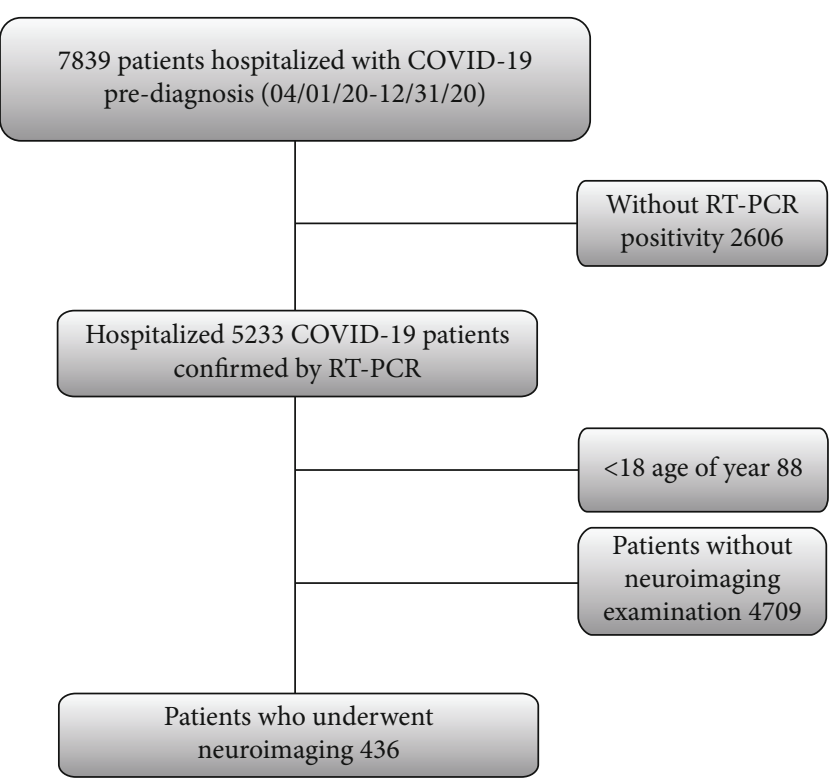

FIgURE 1: Flowchart of patient inclusion. COVID-19: coronavirus disease 2019; RT-PCR: reverse-transcription polymerase chain reaction.

pertaining to the day of neuroimaging (C-reactive protein (CRP), D-dimer level, lymphocyte count, and lactate dehydrogenase $(\mathrm{LDH})$ ) and blood $\mathrm{O}_{2}$ saturation, as well as epidemiological data such as age, sex, and underlying diseases of the patients, were retrieved from the hospital database and recorded.

2.1. Imaging Technique. In all CT examinations, 128- and 16slice multidetector spiral CT scanners (Somatom Definition, Siemens Healthcare, Forchheim, Germany; Optima 520, GE Medical System, USA) were used. In unenhanced brain CT analyses, automatic dosing ( $120 \mathrm{kV} /$ auto-mAs) was achieved on the axial plane from the skull base to the vertex level with slice thicknesses of $5 \mathrm{~mm}$ and $1.25 \mathrm{~mm}$. Coronal and sagittal reconstruction of the images was achieved in the bone and soft tissue algorithm at the slice thickness and interval of 1.5$2 \mathrm{~mm}$. Brain-neck CT angiography analyses were performed with automatic dosing ( $120 \mathrm{kV} /$ auto-mAs) at the slice thickness and interval of $0.625-1.25 \mathrm{~mm}$ on the axial plane, and coronal and sagittal reformatted images with slice thicknesses of 1.5-2 mm were generated. MRI of the brain was performed with $1.5 \mathrm{~T}$ scanners (Magnetom Avanto, Siemens Healthcare, Germany; Optima 360, GE Medical System, USA). In the MRI of the brain, T1-weighted (T1W), T2-weighted (T2W), fluidattenuated inversion recovery (FLAIR), diffusion-weighted imaging (DWI), and susceptibility-weighted imaging (SWI) sequences on the axial plane; T2W and FLAIR on the sagittal plane; T2W sequences on the coronal plane; and where necessary, following administration of $0.1 \mathrm{mmol} / \mathrm{kg}$ gadolinium, T1W sequences on the coronal, sagittal, and axial planes were obtained.

2.2. Statistical Analysis. All statistical analyses were performed using SPSS software ver. 23.0 (SPSS Inc, Chicago IL, USA). The Shapiro-Wilk test was used to test the data 
TABLE 1: Comparison of characteristics of patients with and without abnormal brain imaging result.

\begin{tabular}{|c|c|c|c|c|c|c|c|}
\hline Variables & $\begin{array}{l}\text { Overall } \\
(n=436)\end{array}$ & $\begin{array}{l}\text { Abnormal brain } \\
\text { imaging result } \\
\text { group }(n=46)^{\mathrm{a}}\end{array}$ & $\begin{array}{c}\text { Normal brain } \\
\text { imaging result } \\
\text { group }(n=390)^{\mathrm{b}}\end{array}$ & $p$ value & $\begin{array}{l}\text { Abnormal brain } \\
\text { imaging result } \\
\text { group }(n=189)^{\mathrm{c}}\end{array}$ & $\begin{array}{c}\text { Normal brain } \\
\text { imaging result } \\
\text { group }(n=247)^{\mathrm{d}}\end{array}$ & $p$ value \\
\hline Age (years) & $67(54-76)$ & $69.5(53-79)$ & $66.0(54-75)$ & 0.448 & $71.0(60-79)$ & $63.0(44-73)$ & $<0.001^{*}$ \\
\hline Gender, male & $245(56.2)$ & $32(69.6)$ & $213(54.6)$ & 0.053 & $129(68.3)$ & $116(47.0)$ & $<0.001^{*}$ \\
\hline Hospitalization & $329(75.5)$ & $42(91.3)$ & $287(73.6)$ & $0.008^{*}$ & $160(84.7)$ & $169(68.4)$ & $<0.001^{*}$ \\
\hline Any comorbidity & $294(67.4)$ & $37(80.4)$ & $257(65.9)$ & $0.047^{*}$ & $146(77.2)$ & $148(59.9)$ & $<0.001^{*}$ \\
\hline Hypertension & $230(52.8)$ & $29(63.0)$ & $201(51.5)$ & 0.139 & $113(59.8)$ & $117(47.4)$ & $0.010^{*}$ \\
\hline Previous cerebrovascular disease & $59(13.5)$ & $9(19.6)$ & $50(12.8)$ & 0.206 & $38(20.1)$ & $21(8.5)$ & $<0.001^{*}$ \\
\hline Cardiovascular disease & $89(20.4)$ & $11(23.9)$ & $78(20.0)$ & 0.533 & $47(24.9)$ & $42(17.0)$ & $0.044^{*}$ \\
\hline Renal insufficiency & $32(7.3)$ & $8(17.4)$ & $24(6.2)$ & $0.012^{*}$ & $21(11.1)$ & $11(4.5)$ & $0.008^{*}$ \\
\hline Diabetes mellitus & $93(21.3)$ & $12(26.1)$ & $81(20.8)$ & 0.405 & $44(23.3)$ & $49(19.8)$ & 0.385 \\
\hline COPD & $38(8.7)$ & $1(2.2)$ & $37(9.5)$ & 0.160 & $15(7.9)$ & $23(9.3)$ & 0.614 \\
\hline Any immunosuppression & $10(2.2)$ & $5(10.8)$ & $5(1.3)$ & $<0.001^{*}$ & $7(3.7)$ & $3(1.2)$ & 0.656 \\
\hline Indications for brain imaging & - & - & - & 0.239 & - & - & $<0.001^{*}$ \\
\hline Altered mental status & $247(56.7)$ & $28(60.9)$ & $219(56.2)$ & & $123(65.1)$ & $124(50.2)$ & \\
\hline Syncope & $52(11.9)$ & $3(6.5)$ & $49(12.6)$ & & $10(5.3)$ & $42(17.0)$ & \\
\hline Neurological deficit & $74(17.0)$ & $13(28.3)$ & $61(15.6)$ & & $39(20.6)$ & $35(14.2)$ & \\
\hline Seizure & $9(2.1)$ & $0(0)$ & $9(2.3)$ & & $2(1.1)$ & $7(2.8)$ & \\
\hline Head trauma & $12(2.8)$ & $0(0)$ & $12(3.1)$ & & $3(1.6)$ & $9(3.6)$ & \\
\hline
\end{tabular}

${ }^{*}$ Statistically significant. Values are presented as median (interquartile range) for continuous variables and frequency (percentage) for categorical variables. COPD: chronic obstructive pulmonary disease. ${ }^{\mathrm{a}}$ Group $1,{ }^{\mathrm{b}}$ Group $2,{ }^{\mathrm{c}}$ Group 3, and ${ }^{\mathrm{d}}$ Group 4.

for normality of distribution. Categorical variables were presented as frequencies (percentages) and compared with the chi-square test (or Fisher's exact test, where appropriate). Nonnormally distributed continuous variables were presented as median with interquartile range (IQR, 25th and 75th percentiles) and compared with the Mann-Whitney $U$ test between the groups. After the possible factors were identified by univariate analyses, a logistic regression was performed to ascertain the effect of age, male gender, hospitalization, presence of hypertension, cardiovascular disease, renal insufficiency, previous cerebrovascular disease, and CRP and D-dimer levels on the likelihood that participants have abnormal brain imaging result. The logistic regression model was statistically significant, $\chi^{2}(4)=38.003, p<0.001$. The model fit was assessed using the Hosmer and Lemeshow goodness-of-fit test. The model explained 17.0\% (Nagelkerke $R^{2}$ ) of the variance in having abnormal brain imaging result and correctly classified $89.4 \%$ of cases. For all comparisons, a value of $p<0.05$ was considered statistically significant.

\section{Results}

3.1. Neuroimaging Use and Common Findings. A total of 436 patients were included in this retrospective study: the mean age was $62.4(18-95)$ years, and $245(56.2 \%)$ patients were male. Among all cases, 294 (67.4\%) patients had at least one comorbidity, and hypertension was the most common comorbidity found (230 patients, 52.8\%). The patients undergoing imaging included 329 (75.5\%) hospitalized patients and $107(24.5 \%)$ outpatients due to COVID-19. The most common indications for conducting neuroimaging procedures were altered state of consciousness (247 patients, $56.7 \%$ ), neurological deficit (74 patients, $17 \%$ ), and syncope (52 patients, $11.9 \%$ ) (Table 1 ). The most common used neuroimaging technique was the CT scan of the brain performed in 423 (97\%) patients, which was resorted to for a total of 462 times including repetitions. The mean period of time until neuroimaging analysis was calculated to be 7 days for CT of the brain and 8 days for MRI and DWI. Possible ischemic changes were the most common finding in both brain CT (loss of attenuation in white matter in 135 patients, 31.6\%) and brain MRI (foci of increased signal in T2A and FLAIR sequences in white matter in 123 patients, 28.2\%). In the comparison between group $1(N=46)$ and group 2 $(N=390)$, there was a statistically significant difference in terms of presence of at least one comorbid disease, immunosuppression, and duration of hospitalization $(p=0.047$, $p<0.001$, and $p<0.001$, respectively). No statistically significant difference was found in the comparison of age and sex among groups ( $p=0.448$ and $p=0.801$, respectively). In the comparison between group $3(N=189)$ and group 4 $(N=247)$ with normal neuroimaging findings, there was a statistically significant difference in terms of age, gender, presence of at least one comorbid disease, and neuroimaging indications $(p<0.001, p<0.001, p<0.001$, and $p<$ 0.001 , respectively) (Table 1 ).

3.2. Acute Hemorrhage. Intracranial hemorrhage was detected in $8(1.9 \%)$ patients in brain CT examination, and $4 / 8$ (50\%) of them were gross hemorrhage and subarachnoid hemorrhage. In MR imaging, intracranial hemorrhage was detected in $10(2.3 \%)$ patients with SWI sequence. Of these, 


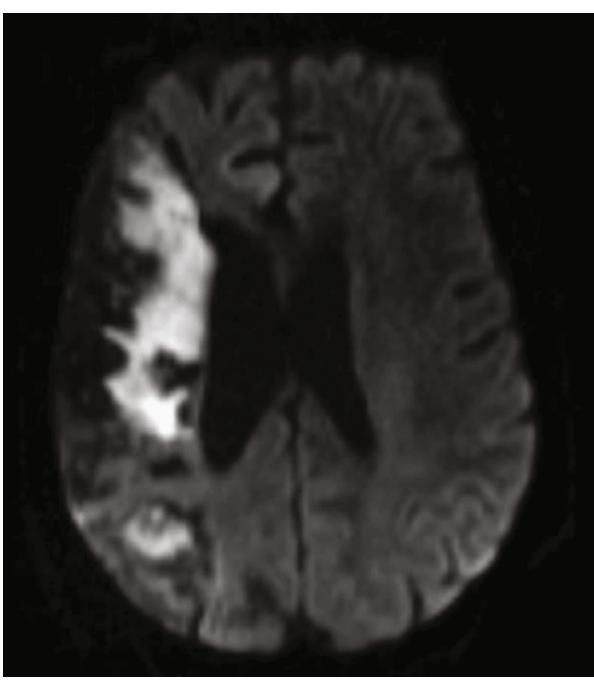

(a)

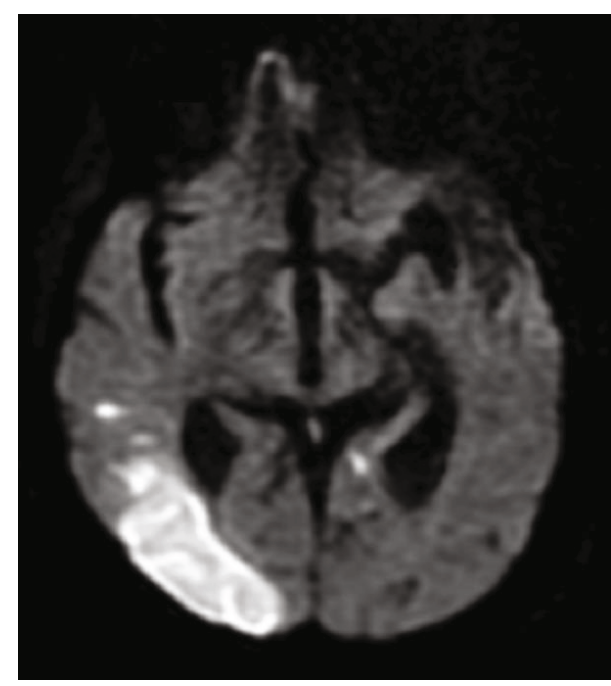

(b)

Figure 2: Samples of acute/subacute infarction in 2 different patients (a, b). (a) On DWI, right middle cerebral artery infarction was observed in a 69-year-old female patient affecting most of the right frontal and parietal lobes. (b) The diffusion restriction was observed in DWI due to large infarction in the posterior cerebral artery irrigation area in the right occipital lobe.
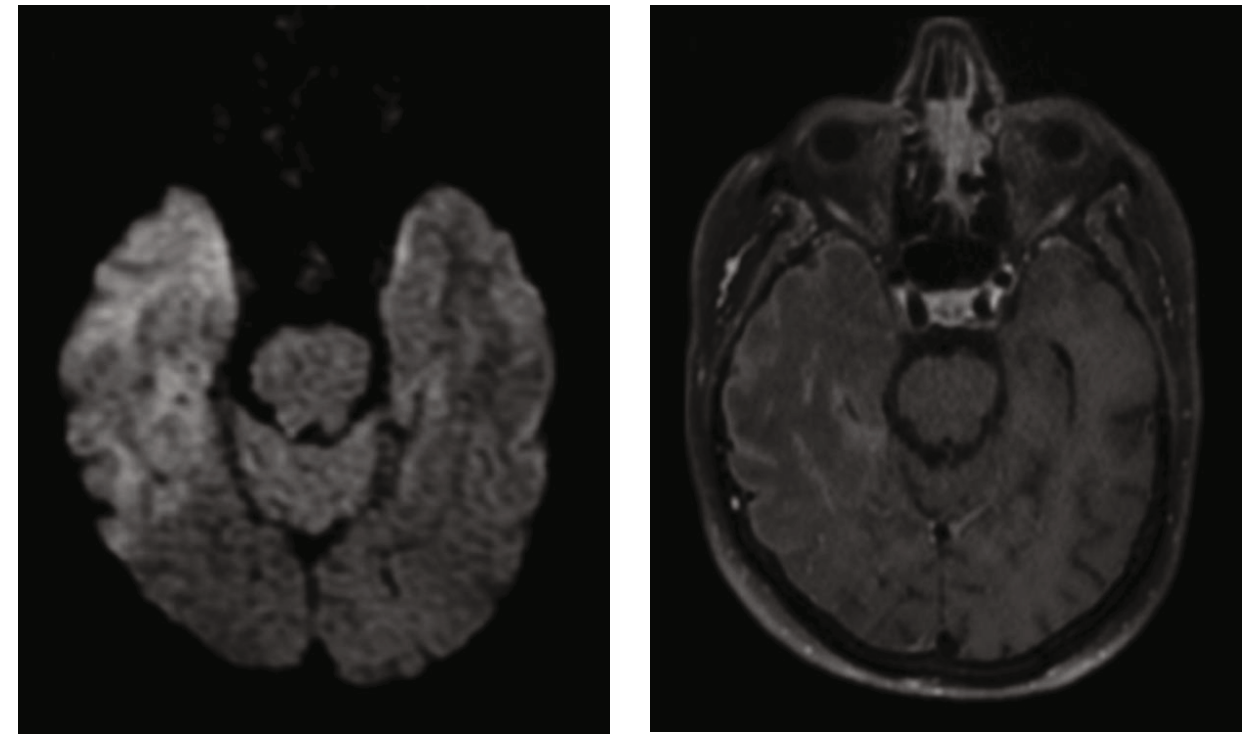

FIGURE 3: Right temporal lobe predominant encephalitis in a 58-year-old male patient (a, b). (a) Moderate diffusion restriction was observed in the DWI image. (b) In the postcontrast T1-weighted image, moderate enhancement in the subcortical and marked enhancement in the subpial subarachnoid were observed.

3/10 (30\%) were gross hemorrhage ( 3 nontraumatic patients with gross hemorrhage on CT) and 7/10 (70\%) were microhemorrhages. Amyloid angiopathy and chronic hypertensive encephalopathy are particularly involved in the etiology of microhemorrhage. In our study, there was no case with findings suggestive of amyloid angiopathy, whereas 7 of 10 patients with microhemorrhage had hypertension. Traumatic cerebral hemorrhage was observed in one patient.

3.3. Acute/Subacute Infarcts and Encephalitis. On DWI, 46 $(28.2 \%)$ of 163 patients had findings consistent with infarction, and 35 (76.1\%) of them were evaluated in favor of ische- mic infarction (Figure 2). In particular, 29 patients with intracranial hemorrhage or infarction were also administered CTA or MRA; of these, 11 (37.9\%) had arterial occlusion and one had venous sinus thrombosis. Since encephalitis was suspected in 3 patients in whom neuroimaging was performed due to altered state of consciousness and confusion, cerebrospinal fluid (CSF) analysis was performed by lumbar puncture, and the findings were consistent with encephalitis (Figure 3). A complete summary of different imaging modalities, their findings, and the average time between the diagnosis of COVID-19 and the first radiological examination is given in Table 2. 
3.4. Laboratory and Other Findings. Duration of hospitalization and mortality rate were significantly higher in group 1 compared with group $2(p=0.039$ and $p=0.014$, respectively). Serum levels of CRP and D-dimer were significantly higher in group $1(N=46)$ compared to group $2(N=390)$, while serum lymphocyte count and $\mathrm{SpO}_{2}$ values were significantly lower $(p=0.014, p<0.001, p<0.001$, and $p=0.002$, respectively) (Figure 4). In multivariate analysis, there was a $67 \%$ decrease in the likelihood of abnormal neuroimaging findings for each unit increase in lymphocyte count (odds ratio (OR) 0.33 (0.16-0.69)). Patients with renal insufficiency were 3.03 times more likely to have an abnormal brain imaging result (Table 3). The optimum cut-off values of serum D-dimer, CRP, and LDH are given in Table 4 to predict acute/subacute infarction. Compared with the non-COVID acute/subacute thromboembolic patient group $(N=40)$, serum D-dimer and CRP levels were found to be significantly higher and lymphocyte counts were significantly lower in COVID-19-positive acute/subacute thromboembolism cases ( $p=0.02,0.005$, and $<0.001$, respectively) (Table 5) (Figure 5).

\section{Discussion}

Neuroimaging in patients with COVID-19 is usually performed due to changes in mental status and headache, which may develop secondary to hypoxemia and respiratory distress, as well as neurological deficits, syncope, convulsions, or trauma. The most common cause of neuroimaging was altered consciousness at a rate of $68 \%$ (214/329 inpatients) in hospitalized patients and $56.7 \%$ in the all study population. The second most common reason for neuroimaging was focal neurological deficit, with a rate of $17 \%$ in the cohort and $13.7 \%$ (45/329 inpatients) among hospitalized patients. What is striking here is that the incidence of altered state of consciousness was higher in inpatients and that of focal neurological deficit was higher in outpatients compared with in the study cohort. This can be explained by altered mental status that may develop secondary to respiratory distress and low oxygen saturation in blood in hospitalized patients. Radmanesh et al. reported that the 3 most common clinical indications for neuroimaging were altered mental status (42.1\%), syncope/fall (32.6\%), and focal neurological deficit (12.4\%) [15].

The most common abnormal finding in the neuroimaging results of 436 patients included in our study cohort was nonspecific white matter changes evaluated in 189 (43.3\%) patients. In the group with nonspecific white matter changes, mean patient age and duration of hospitalization, presence of comorbid disease, and mortality rate were found to be significantly higher than in the group of patients without abnormal neuroimaging findings. One of the reasons for this may be that the rate of presence of accompanying comorbid diseases was significantly higher in these patients compared with the group without abnormal findings [16]. In addition, presence of comorbid diseases and mortality rates were significantly higher in the group of patients with acute/subacute infarction and hemorrhage than in those without abnormal neuroimaging findings. In a study conducted at a single center in the
TABLE 2: Brain imaging results of 436 COVID-19 patients.

\begin{tabular}{|c|c|}
\hline Variable & Value \\
\hline \multicolumn{2}{|l|}{ Brain CT $(n=423)$} \\
\hline Time* of first CT exam (days) & $7(6-9)$ \\
\hline No abnormality & $265(62.7)$ \\
\hline Infarction & $15(3.5)$ \\
\hline Intracranial hemorrhage & $8(1.9)$ \\
\hline Nonspecific white matter changes & $135(31.9)$ \\
\hline \multicolumn{2}{|l|}{ Brain MRI $(n=243)$} \\
\hline Time* of first MR exam (days) & $8(6-10)$ \\
\hline Intracranial hemorrhage (for SWI) & $10(4.1)$ \\
\hline Nonspecific white matter changes & $123(50.6)$ \\
\hline \multicolumn{2}{|c|}{ Location of nonspecific white matter changes $(n=123)$} \\
\hline Frontal lobe & $78(63.4)$ \\
\hline Parietal lobe & $68(55.3)$ \\
\hline Temporal lobe & $32(26.0)$ \\
\hline Occipital lobe & $25(20.3)$ \\
\hline Pons-mesencephalon & $19(15.4)$ \\
\hline Basal ganglia & $32(26.0)$ \\
\hline Corpus callosum & $12(9.8)$ \\
\hline Periventricular & $41(33.3)$ \\
\hline Cerebellar & $7(5.7)$ \\
\hline \multicolumn{2}{|l|}{ CTA $(n=18)$} \\
\hline Occlusion & $8(44.4)$ \\
\hline Right MCA & $5(62.5)$ \\
\hline Left MCA & $1(12.5)$ \\
\hline Left PCA & $2(25.0)$ \\
\hline \multicolumn{2}{|l|}{$\operatorname{MRA}(n=11)$} \\
\hline Occlusion & $5(45.5)$ \\
\hline Right MCA & $3(60.0)$ \\
\hline Right PCA & $1(20.0)$ \\
\hline Venous sinus thrombosis & $1(20.0)$ \\
\hline \multicolumn{2}{|l|}{ DWI $(n=163)$} \\
\hline Time* of first diffusion exam (days) & $8(6-10)$ \\
\hline Infarction & $46(28.2)$ \\
\hline \multicolumn{2}{|l|}{ Infarction site } \\
\hline Right hemisphere & $13(28.3)$ \\
\hline Left hemisphere & $12(26.1)$ \\
\hline Both hemisphere & $18(39.1)$ \\
\hline Cerebellum & $3(6.5)$ \\
\hline
\end{tabular}

${ }^{*}$ Interval between diagnosis of COVID-19 and first brain imaging exam.

USA which included 242 patients, it was reported that the mortality rate and the likelihood of developing infarction were significantly higher in the group with white matter microangiopathy than in the group without abnormal findings [15].

In this study, 46 patients had acute/subacute infarction, and the overall incidence in the cohort was $10.5 \%$. In a recently published meta-analysis, it was reported that the rate of acute/subacute infarction observed in neuroimaging procedures ranged from $5.4 \%$ to $23.3 \%$ in patients with 


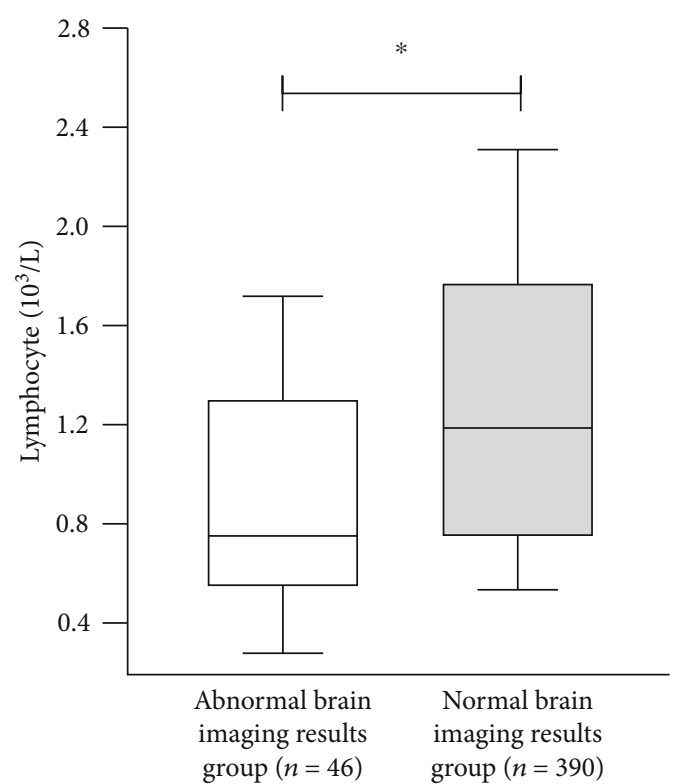

(a)

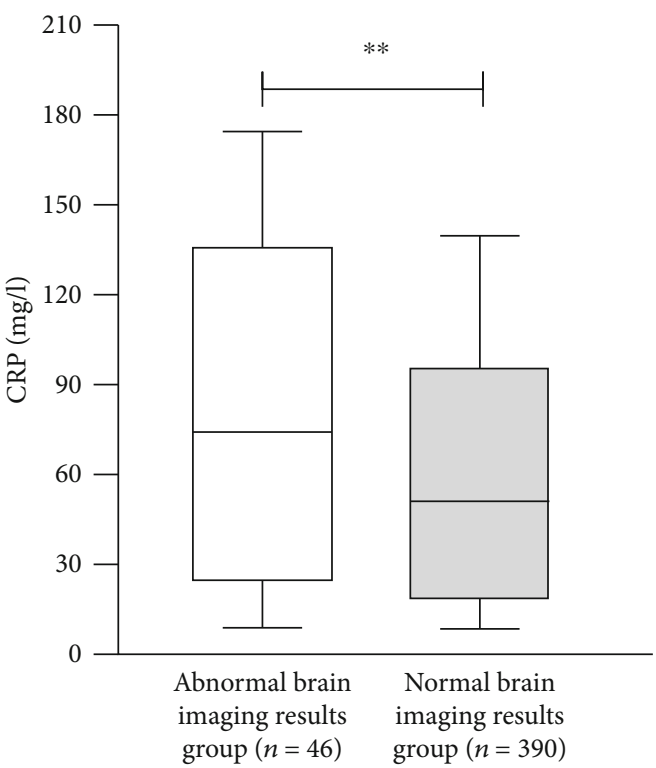

(c)

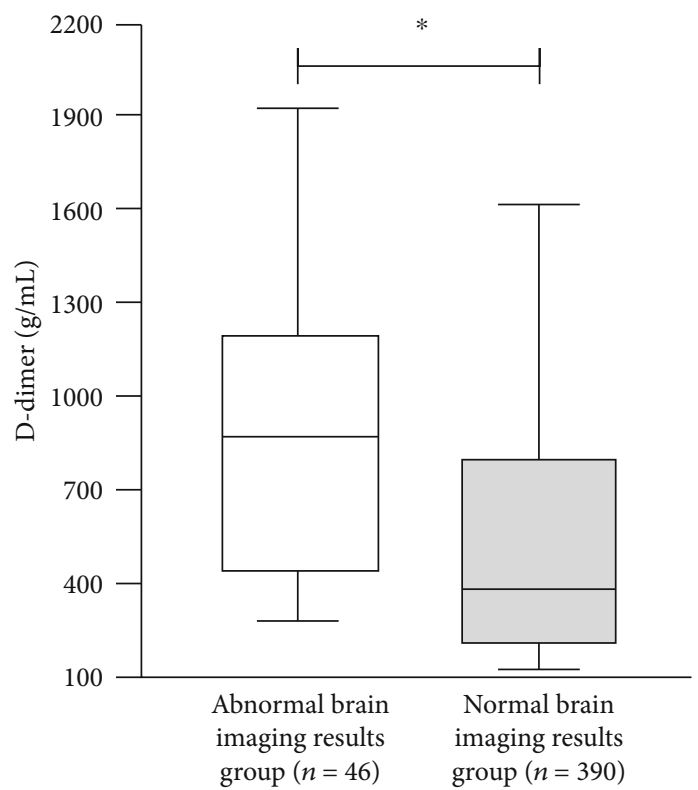

(b)

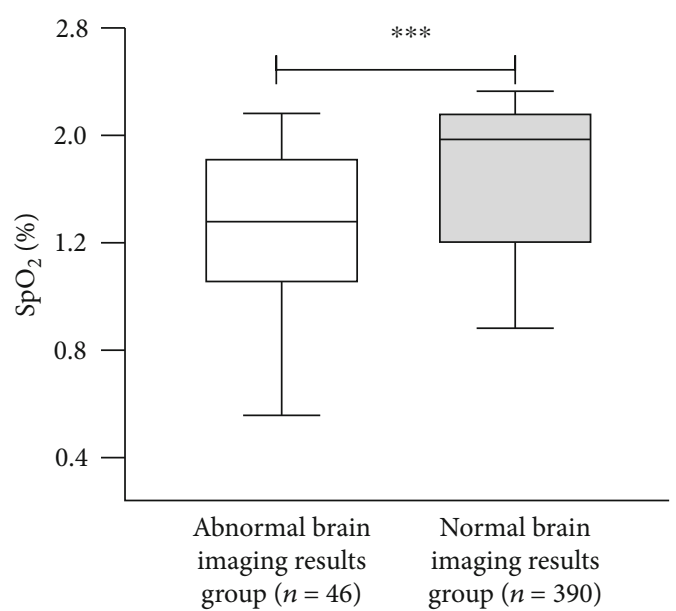

(d)

FIgURe 4: Continued. 


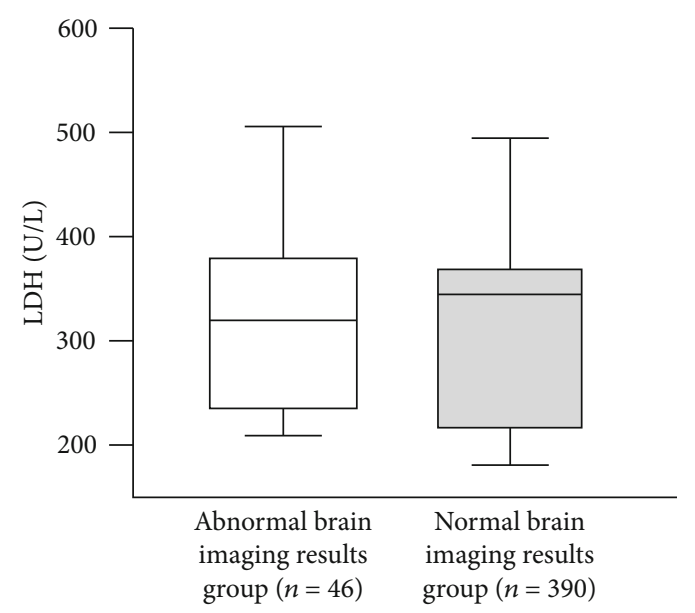

(e)

FIGURE 4: Differences in baseline characteristics of patients according to study groups: (a) lymphocyte, (b) D-dimer, (c) CRP, (d) SpO ${ }_{2}$, and (e) LDH. ${ }^{*} p<0.001,{ }^{* *} p=0.014,{ }^{* * *} p=0.002$, and $p=0.067$. Data are presented with the Tukey box-and-whisker plot, where the middle line represents the median and the box represents the IQR (the 25th and 75th percentiles).

TABLE 3: Results of multivariate logistic regression model in predicting the presence of acute/subacute thromboembolism in COVID-19 patients.

\begin{tabular}{lccc}
\hline Variable & OR & $95 \%$ CI & $p$ value \\
\hline Age & 0.981 & $0.960-1.002$ & 0.083 \\
Male gender & 1.673 & $0.802-3.492$ & 0.170 \\
COPD & 0.169 & $0.022-1.322$ & 0.090 \\
Diabetes mellitus & 1.986 & $0.824-3.542$ & 0.079 \\
Hypertension & 1.943 & $0.938-4.026$ & 0.074 \\
Renal insufficiency & 3.030 & $1.108-8.292$ & $0.031^{*}$ \\
CRP & 0.997 & $0.993-1.002$ & 0.289 \\
D-dimer & 1.000 & $0.984-1.003$ & $0.002^{*}$ \\
LDH & 1.004 & $0998-1.007$ & 0.341 \\
Lymphocyte & 0.336 & $0.162-0.696$ & $0.003^{*}$ \\
SpO 2 & 0.941 & $0.885-1.002$ & 0.056 \\
\hline
\end{tabular}

*Statistically significant. OR: odds ratio; Cl: confidence interval; CRP: Creactive protein; COPD: chronic obstructive pulmonary disease.

COVID-19 [17]. Jain et al. reported the rate of acute/subacute infarction as $7.7 \%$ in their cohort of 454 patients [18]. Low molecular weight heparin (LMWH) was used in all patients with COVID-19 who received inpatient treatment in our center, if there were no contraindications. This may have reduced the likelihood of possible thromboembolic events.

The rate of microhemorrhage and/or intracranial hemorrhage in our study was $3.4 \%$, which was lower than the rates reported by Sawlani et al. (9\%) and Yoon et al. (6\%) $[19,20]$. The most important reasons for these differences may be associated with the nonhomogeneity of the selected cohorts (accompanying comorbidity and other demographic characteristics) and the choice of imaging modality. In the metaanalysis published by Choi and Lee, which included 21 articles/case reports, it was reported that the incidences of microhemorrhage, infarction and encephalitis in COVID19 patients were significantly higher when mainly MRI was used for neuroimaging compared to studies using CT alone or CT-weighted neuroimaging [17].

In the present study, D-dimer level was found to be significantly higher in 46 COVID-19-positive patients with acute thromboembolic events. In addition, in the comparison with the group of 40 cases with non-COVID acute/subacute thromboembolism, it was found that the D-dimer and CRP levels were significantly higher and the lymphocyte count was significantly lower in the COVID-19-positive group. It has been reported that viral infections, and in particular SARS-CoV-2, are associated with an increase in prothrombotic events such as ischemic stroke [21-23]. It has been claimed that viral infections lead to an increase in procoagulant markers, leading to thrombosis as well as disseminated intravascular coagulation and hemorrhagic events [23]. In a study published by Beyrouti et al., significantly high Ddimer levels were observed in all 6 patients who were followed up with the diagnosis of COVID-19 and who experienced acute thromboembolic events during this period [24]. Kremer et al. reported an elevated D-dimer level $(>1000 \mu \mathrm{g} / \mathrm{L})$ in 10 of 11 patients with acute thromboembolic events [25].

Encephalitis cases presenting with quite different rates and radioclinical data caused by SARS-CoV-2 have been reported. In our study, there were clinical, laboratory, and radiological findings consistent with encephalitis in 3 $(0.7 \%)$ patients. Two of the patients had predominant right frontoparietal and temporal lobe and left frontobasal lobe involvement. Significant increase in signal was observed in T2W and FLAIR sequences, and patchy diffusion restriction was observed in DWI. In postcontrast T1W images, contrast enhancement was nodular in the white matter and linear in the meningeal-pial region. In a recently published metaanalysis, it was reported that the incidence of encephalitis among cohort patients ranged from $1.9 \%$ to $4.7 \%$ [17]. 
TABLE 4: Comparison of area under curve (AUC) to predict the acute/subacute thromboembolism in COVID-19 patients.

\begin{tabular}{|c|c|c|c|c|c|}
\hline & $\operatorname{AUC}(95 \% \mathrm{CI})$ & Cut-off & Sensitivity & Specificity & $p$ value \\
\hline CRP (mg/L) & $0.557(0.465-0.648)$ & 8.4 & 0.926 & 0.691 & $<0.230$ \\
\hline D-dimer $(\mathrm{ng} / \mathrm{mL})$ & $0.828(0.782-0.875)$ & 756 & 0.829 & 0.739 & $<0.001$ \\
\hline $\mathrm{LDH}(\mathrm{U} / \mathrm{L})$ & $0.595(0.519-0.672)$ & 0.56 & 0.667 & 0.392 & 0.045 \\
\hline
\end{tabular}

CRP: C-reactive protein; LDH: lactate dehydrogenase.

TABLE 5: Demographic and laboratory data in acute/subacute thromboembolism cases.

\begin{tabular}{lccc}
\hline Variables & COVID-19-positive group $(n=46)$ & Non-COVID group $(n=40)$ & $p$ value \\
\hline Age (years) & $61.1(51.0-79.0)$ & $66.2(54.0-86.0)$ & 0.215 \\
Gender, male (\%) & $32(69.6)$ & $12(30.0)$ & $0.003^{*}$ \\
Any comorbidity (\%) & $37(80.4)$ & $31(77.5)$ & 0.323 \\
Mortality (\%) & $14(30.4)$ & 31.7 & 0.194 \\
CRP level (mg/L) & 78.7 & 306.2 & $0.005^{*}$ \\
LDH level (U/L) & 362.6 & 631.8 & 0.098 \\
D-dimer level $(\mathrm{ng} / \mathrm{mL})$ & 2506.8 & 2.55 & $0.020^{*}$ \\
Lymphocyte $\left(10^{3} / \mu \mathrm{L}\right)$ & 1.23 & & $<0.001^{*}$ \\
\hline
\end{tabular}

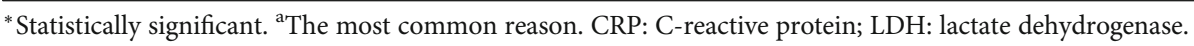

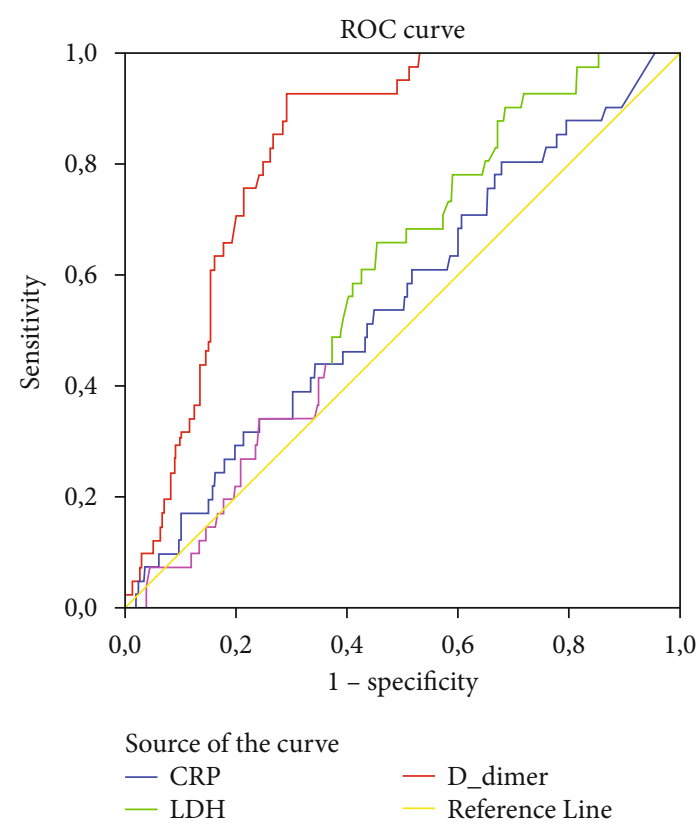

FIGURE 5: ROC curves for CRP, D-dimer, and LDH in predicting acute/subacute infarction in COVID-19 patients.

One patient had transient mild encephalopathy and a wellcircumscribed lesion localized in the middle part of the splenium of the corpus callosum on MRI. It was observed that the lesion had marked diffusion limitation and disappeared on the follow-up DWIs, and it was primarily evaluated as a cytotoxic lesion of the corpus callosum (CLOCC).

In conclusion, in this study, we aimed to share the neuroimaging findings of patients who developed neurological symptoms during their follow-up due to COVID-19 and to reveal the relationship between acute thromboembolic events and some laboratory parameters. Patients with COVID-19-positive acute/subacute infarcts have significantly higher D-dimer levels compared to patients with COVID-19-negative acute/subacute infarcts. This supports that viral infections in general, and SARS-CoV-2 in particular, increase the risk of acute thromboembolism by increasing procoagulant markers (inflammatory or autoimmune). We believe that the risk of acute thromboembolism increases significantly in COVID-19 patients when serum $\mathrm{D}$-dimer level is above $756 \mathrm{ng} / \mathrm{dL}$, and detailed neurological evaluation and neuroimaging when necessary in these patients will be beneficial.

First of all, since CT was used predominantly in our study, some pathologies that could be detected by MR, and especially the changes in the cortex or white matter, might not have been detected. In addition, the necessary neuroimaging could not be performed in some of the patients who were being treated under intensive care conditions due to the failure to provide appropriate technical requirements.

\section{Data Availability}

The data used to support the findings of this study are available from the corresponding author upon request.

\section{Ethical Approval}

The study was conducted according to the guidelines of the Declaration of Helsinki, and the approval of the study was granted by the Institutional Ethics Committee (Gazi Yaşargil Training and Research Hospital, decision no. 587/2020). 


\section{Consent}

As this study is retrospective, the informed consent form was waived.

\section{Conflicts of Interest}

The authors declare no conflicts of interest.

\section{Authors' Contributions}

Conceptualization was performed by Kavak, Seyhmus. Data curation was performed by Kavak, Seyhmus; Yildirim, Mehmet Serdar; Altındag, Rojhat; Mertsoy, Yilmaz; Alakus, Mehmet Fuat; Guleken, Mehmet Diyaddin; and Kaya, Safak. Formal analysis was performed by Kavak, Seyhmus. Investigation was performed by Kavak, Seyhmus and Kaya, Safak. Methodology was performed by Kavak, Seyhmus. Project administration was performed by Kavak, Seyhmus. Kavak, Seyhmus and Kaya, Safak were responsible for the resources. Kavak, Seyhmus was responsible for the software. Supervision was performed by Kaya, Safak. Validation was performed by Kavak, Seyhmus. Visualization was performed by Kavak, Seyhmus. Writing of original draft was performed by Kavak, Seyhmus. Writing, review, and editing were performed by Kavak, Seyhmus and Kaya, Safak.

\section{Acknowledgments}

Thanks are due to radiology experts Dr. Recai Duymus and Dr. Aydin Aslan.

\section{References}

[1] World Health Organization, Director-General's remarks at the media briefing on 2019-nCoV on 11 February 2020, 2020, http://www.who.int/dg/speeches/detail/who-director-generals-remarks-at-the-media-briefing-on-2019-ncov.

[2] Centers for Disease Control and Prevention, Novel coronavirus, Information for Healthcare Professionals, Wuhan, China, 2019, https://www.cdc.gov/coronavirus/2019-nCoV/hcp/ index.html-2020.

[3] H. Xia and E. Lazartigues, "Angiotensin-converting enzyme 2 in the brain: properties and future directions," Journal of Neurochemistry, vol. 107, no. 6, pp. 1482-1494, 2008.

[4] J. Netland, D. K. Meyerholz, S. Moore, M. Cassell, and S. Perlman, "Severe acute respiratory syndrome coronavirus infection causes neuronal death in the absence of encephalitis in mice transgenic for human ACE2," Journal of Virology, vol. 82, no. 15, pp. 7264-7275, 2008.

[5] M. Dubé, A. Le Coupanec, A. H. M. Wong, J. M. Rini, M. Desforges, and P. J. Talbot, "Axonal transport enables neuron-to-neuron propagation of human coronavirus OC43," Journal of Virology, vol. 92, no. 17, 2018.

[6] E. B. Milbrandt, M. C. Reade, and M. Lee, "Prevalence and significance of coagulation abnormalities in community-acquired pneumonia," Molecular Medicine, vol. 15, no. 11-12, pp. 438445, 2009.

[7] F. A. Klok, M. J. H. A. Kruip, N. J. M. van der Meer et al., "Incidence of thrombotic complications in critically ill ICU patients with COVID-19," Thrombosis Research, vol. 191, pp. 145-147, 2020.

[8] T. J. Oxley, J. Mocco, S. Majidi et al., "Large-vessel stroke as a presenting feature of COVID-19 in the young," The New England Journal of Medicine, vol. 382, no. 20, article e60, 2020.

[9] F. Zhou, T. Yu, R. Du et al., "Clinical course and risk factors for mortality of adult inpatients with COVID-19 in Wuhan, China: a retrospective cohort study," Lancet, vol. 395, no. 10229, pp. 1054-1062, 2020.

[10] R. P. MUNHOZ, J. L. PEDROSO, F. A. NASCIMENTO et al., "Neurological complications in patients with SARS-CoV-2 infection: a systematic review," Arquivos de Neuro-Psiquiatria, vol. 78, no. 5, pp. 290-300, 2020.

[11] J. E. Kim, J. H. Heo, H. O. Kim et al., "Neurological complications during treatment of Middle East respiratory syndrome," Journal of Clinical Neurology, vol. 13, no. 3, pp. 227-233, 2017.

[12] L. K. Tsai, S. T. Hsieh, C. C. Chao et al., "Neuromuscular disorders in severe acute respiratory syndrome," Archives of Neurology, vol. 61, no. 11, pp. 1669-1673, 2004.

[13] Y. M. Arabi, A. Harthi, J. Hussein et al., "Severe neurologic syndrome associated with Middle East respiratory syndrome corona virus (MERS-CoV)," Infection, vol. 43, no. 4, pp. 495501, 2015.

[14] K.-K. Lau, W.-C. Yu, C.-M. Chu, S.-T. Lau, B. Sheng, and K.-Y. Yuen, "Possible central nervous system infection by SARS coronavirus," Emerging Infectious Diseases, vol. 10, no. 2, pp. 342-344, 2004.

[15] A. Radmanesh, E. Raz, E. Zan, A. Derman, and M. Kaminetzky, "Brain imaging use and findings in COVID19: a single academic center experience in the epicenter of disease in the United States," AJNR. American Journal of Neuroradiology, vol. 41, no. 7, pp. 1179-1183, 2020.

[16] E. J. van Dijk, M. M. Breteler, R. Schmidt et al., "The association between blood pressure, hypertension, and cerebral white matter lesions," Hypertension, vol. 44, no. 5, pp. 625-630, 2004.

[17] Y. Choi and M. K. Lee, "Neuroimaging findings of brain MRI and CT in patients with COVID-19: a systematic review and meta-analysis," European Journal of Radiology, vol. 133, p. 109393, 2020.

[18] R. Jain, M. Young, S. Dogra et al., "COVID-19 related neuroimaging findings: a signal of thromboembolic complications and a strong prognostic marker of poor patient outcome," Journal of the Neurological Sciences, vol. 414, p. 116923, 2020.

[19] V. Sawlani, S. Scotton, K. Nader et al., "COVID-19-related intracranial imaging findings: a large single-centre experience," Clinical Radiology, vol. 76, no. 2, pp. 108-116, 2021.

[20] B. C. Yoon, K. Buch, M. Lang et al., "Clinical and neuroimaging correlation in patients with COVID-19," AJNR. American Journal of Neuroradiology, vol. 41, no. 10, pp. 1791-1796, 2020.

[21] J. Helms, C. Tacquard, F. Severac, I. Leonard-Lorant, M. Ohana, and X. Delabranche, "High risk of thrombosis in patients with severe SARS-CoV-2 infection: a multicenter prospective cohort study," Intensive Care Medicine, vol. 46, no. 6, pp. 1089-1098, 2020.

[22] M. A. Nagel, R. Mahalingam, R. J. Cohrs, and D. Gilden, "Virus vasculopathy and stroke: an under-recognized cause and treatment target," Infectious Disorders Drug Targets, vol. 10, no. 2, pp. 105-111, 2010. 
[23] S. Subramaniam and I. Scharrer, "Procoagulant activity during viral infections," Frontiers in Bioscience, vol. 23, pp. 10601081, 2018.

[24] R. Beyrouti, M. E. Adams, L. Benjamin et al., "Characteristics of ischaemic stroke associated with COVID-19," Journal of Neurology, Neurosurgery, and Psychiatry, vol. 91, no. 8, pp. 889-891, 2020.

[25] S. Kremer, F. Lersy, M. Anheim et al., "Neurologic and neuroimaging findings in patients with COVID-19: a retrospective multicenter study," Neurology, vol. 95, no. 13, pp. e1868e1882, 2020. 